\title{
XVIII Festival Internacional de Música Contemporánea
}

\author{
Por \\ Fernanda Ortega Sáenz \\ Departamento de Música \\ Universidad Metropolitana de Ciencias de la Educación, Chile \\ lfortega@uc.cl
}

Entre el lunes 15 y el viernes 18 de enero de 2018 se desarrolló el XVIII Festival Internacional de Música Contemporánea que organiza el Departamento de Música de la Facultad de Artes de la Universidad de Chile.

Una nueva versión del festival, la decimoctava, se realizó en enero en la sala Isidora Zegers. Se inició con palabras de la Decana, Sra. Clara Luz Cárdenas Squella, y de Wilson Padilla Véliz, Director del nuevo Departamento de Música (DMUS), luego de la separación y creación en 2017 del Departamento de Sonido de esta casa de estudios. La inauguración -muy breve, a diferencia de la considerable extensión habitual- no contó con homenaje, centrándose en mencionar la falta del financiamiento que anteriormente otorgó el Consejo Nacional de la Cultura y las Artes. Este hecho marcó el sello de esta edición, más austera, sin intérpretes ni conjuntos extranjeros invitados, pero con un decidido esfuerzo de parte de la Facultad de Artes, del Decanato, su Dirección Económica y de los organizadores, por sacar adelante el festival.

Un Comité Seleccionador integrado por académicos del DMUS escogió treinta y siete obras, con dieciséis estrenos absolutos -doblando la cifra de años anteriores-, y ocho estrenos en Chile. Entre el total, escuchamos veintiocho obras de compositores chilenos, un aumento, en oposición a la disminución de obras latinoamericanas y europeas, siete y dos, respectivamente.

El año de composición promedio también presentó una variación considerable. Así, prácticamente no hubo obras referentes de los diversos períodos del siglo XX, con excepción de tres de ellas. La más antigua fue de 1939, de Karl Amadeus Hartmann, interpretada por la Orquesta Sinfónica Nacional de Chile (OSNCH), sumada a dos obras de 1964 y 1968, en recuerdo al Premio Nacional de Artes Musicales 2008, Miguel Letelier Valdés, fallecido recientemente ${ }^{1}$. No hubo ni una sola obra compuesta entre la década del setenta e inicios del siglo XXI. Por el contrario, presenciamos treinta y cuatro obras del presente siglo, de estas, once fueron compuestas en 2017.

${ }^{1}$ Revisar In Memoriam en Revista Musical Chilena LXXI/227 (enero-junio, 2017), p. 168.

Revista Musical Chilena, Año LXXII, enero-junio, 2018, Nº 229, pp. 151-156 
Respecto de los intérpretes nacionales o residentes, participaron doce conjuntos estables, incluida la OSNCH. Presente por segundo año estuvo el Ensamble de Profesores DMUS bajo la dirección de Andrés Maupoint Álvarez. Para flauta, clarinete, dos violines, viola, cello, piano y percusión, presentaron Multiverso (2016), del joven Aníbal Vidal. Puntillista, construida con breves gestos combinados con texturas de momentos horizontales y pausas bien logradas, destacó por su buena factura y uso del ensamble. De Nicolás Cortés Ahumada, otro joven compositor, presentaron El hilo negro del destino (2017) para flauta, clarinete, saxofón, violín, cello, contrabajo y piano. Enérgica, con movimiento constante organizado entre los diferentes instrumentos, en gestualidades cíclicas de velocidades y energías cambiantes, generó momentos de intensidad continua. Interesante y bien lograda propuesta.

El Cuarteto Surkos siempre presente, interpretó Grilletes y Cadenas (2016), obra en cuatro pequeños movimientos de René Silva Ponce. Iniciada en notas tenidas y luego pasajes arpegiados, combinó diversas texturas en bloques sonoros que generaron cierta tensión expresiva, derivando en pausas bien logradas. Por su parte, el ensamble Tricahue presentó Four trío estudes (2010) para saxofón alto, saxofón soprano, marimba y chinchín, del holandés Guillermo Lago. Original para saxofones y acordeón, mostraron una adaptación con percusión, opción del ensamble. A base de motivos en paralelismos, repetitivos y minimalistas, los estudios intercalaron lo rítmico-movido con lo melódico-calmo, siempre dentro de lo tonal.

Agrupación debutante que esperamos siga de manera estable por sus destacados intérpretes fue el Ensamble Zero dirigido por Andrés Maupoint, con Le tombeau d'une etoile (2016) de su autoría, para flauta en sol, cello, vibráfono y piano. Centrada en el piano, sobre la base de motivos utilizados variadamente y con movimiento perpetuo interior, elaborado en paralelismos y texturas, construyó enérgicamente sus clímax. Ejecutantes reunidos para esta oportunidad interpretaron Tejidos al viento (2015), para trompeta, cello, cajón peruano y piano del peruano Abraham Padilla, inspirada en la cultura precolombina Wari. Si bien utilizó una atractiva conformación instrumental, las diferentes líneas y timbres no alcanzaron a empastarse del todo, funcionando algo separadamente.

En cuanto a ensambles de vientos, la orquesta Illawara dirigida por Wilson Padilla estuvo con Bajo la alborada, una voz... (2015), de la única mujer compositora seleccionada, la chilena Tamara Miller Ilabaca. Obra colorística, interesante, generó espacios sonoros combinando figuras rápidas y melismáticas con sonoridades "nocturnas" y momentos más estáticos. El octeto de saxofones Sur del Mundo estuvo con El opuesto al revés (2017), del licenciado de esta universidad Marcos Contreras, quien participó como intérprete. De lenguaje tonal que comparte la estética de ciertos repertorios de música incidental y de jazz, alternó secciones rítmicas de notas cortas con pasajes melódicos, secciones de repeticiones y movimiento constante.

Por las percusiones, el ensamble Xilos de Valparaíso dirigido por Félix Carbone Kind presentó La caída de Hannush Achtel (2007) del también porteño Jaime Frez Rosales. Para 5 percusionistas, inspirada en los indígenas Yamanas del Sur de Chile. Introducida por una voz en off describiendo la motivación extramusical, fue ejecutada con atuendo e iluminación vinculados a la temática. De momentos frenéticos y otros más pausados, combinó instrumentos de diverso color y timbre, lo que le dio mayor textura.

El Ensamble Compañía de Música Contemporánea que dirige Carlos Valenzuela Ramos participó con cuatro obras de jóvenes compositores chilenos, si bien no incluyeron estrenos. De Francisco Rañilao Elizondo presentaron La metamorfosis de la Mariposa (2015), con José Luis Urquieta como oboe solista en una muy buena interpretación, junto con flauta, clarinete, violín, viola, cello, piano y percusión. Con una línea de oboe melismática y gestual, en interacción con el ensamble que actuó como fondo de color y resonancia, en variaciones más o menos sutiles de sonoridades y motivos recurrentes. De Christian Vásquez Miranda, mostraron Triángulo X (2017), para flauta, clarinete, violín, cello, piano y percusión. Utilizó 
dinámicas pianissimo con amplios crescendos, a base de resonancias del piano y la percusión, bien combinados con los instrumentos melódicos en sonoridades y técnicas contemporáneas, generando un carácter tenso y de cierta aspereza sonora que reforzó la intensidad.

Luego, La Caja de Tensión (2016) para clarinete, violín, viola, cello, piano y percusión, de Juan Manuel Quinteros Saavedra, desarrolló distintos planos gestuales que le dieron a la obra más profundidad, con riqueza tímbrica en donde la percusión fue aporte. Con variedad rítmica y métrica en constantes quiebres, logró vivacidad y energía interna que mantuvieron al auditor cautivado. Finalmente, Re(vb)ela (2015) para flauta, clarinete, violín, viola, cello, piano y percusión de Julio Torres, pianista del ensamble. Con abundancia de sonidos basados en el piano, en un continuo sonoro, tuvo pasajes repetitivos y otros con quiebres y gestos breves. Resultó extensa en su eterno retorno de varias secciones, lo que la hizo perder efectividad.

Ensamble que volvió a participar fue el Taller de Música Contemporánea dirigido por Pablo Aranda Rojas, comenzando con Akira (2017) para violín, cello, piano y vibráfono, del académico del DMUS, Antonio Carvallo Pinto. Organizada en secciones, estáticas o dinámicas, activadas por un motivo inicial insistente, logró disolución de la sensación temporal al interior de cada sección, con sustento en la textura generada por la combinación instrumental. Del aún estudiante de la Universidad de Chile, Diego González Orellana, interpretaron Entre (2015-16), para flauta, clarinete, violín, cello, piano y percusión, una de las interesantes propuestas de esta edición. Buscando ir algo más allá tanto en la forma como en el material, construyó un extenso discurso estructurado de momentos que desembocaron unos sobre otros, combinando pasajes ruidísticos, técnicas extendidas, momentos frenéticos y pausas sonoras con armonías que atraparon, transmitiendo fresca energía.

Para gran ensamble, escuchamos Permiso, Entrada, Ruedas y Ch'alla (2017), del joven ariqueño Guillermo Andrade, por la Orquesta Andina PUCV y cuarteto de Tarkas bajo dirección de Félix Cárdenas Vargas. Incluyó variedad de aerófonos andinos así como charango, tiple, guitarras, bajo eléctrico y percusiones. En tres movimientos de interesante combinación sonora y aires de carnaval nortino, con insistencia en dinámicas fuertes y tuttis, estuvo algo saturado el volumen del bajo y percusiones, perdiéndose por momentos el equilibrio sonoro.

Revisando las conformaciones más pequeñas, el dúo Orellana \& Orlandini estuvo con Paisaje en seis del académico del DMUS Edgardo Cantón Aguirre. Original para guitarra sola (2012), presentada en adaptación de Francisco González (2016), idiomática para el buen trabajo del dúo. Con armonías tonales en escritura motívica repetitiva, jugó con articulaciones, acentos y combinaciones rítmicas. Otro dúo fue $(L / P)^{*} d=j$ (los pasos) (2016), para fagot y marimba del también académico del DMUS Mario Mora López, donde el fagot llevó una suerte de fraseo melódico construido con abundancia de intervalos extensos mientras la marimba mantuvo una participación más segmentada, transitando a secciones de similitud rítmica. Resultaron algo separadas las líneas instrumentales, combinándose solo por momentos. Queda la duda si esta obra es realmente idiomática para ambos instrumentos.

Desde La Serena, José Luis Urquieta y Leonardo Cuevas estuvieron con Tres piezas para dos oboes (2017) del chileno Francisco Silva Cárdenas. La primera, en movimientos descendentes que recordaron a Ligeti, obsesivos y enérgicos, para luego en la segunda tocar sobre el arpa del piano, lo que bajó un poco la intensidad, ya que las resonancias no alcanzaron a envolver el espacio de la sala. La tercera pieza retomó el diálogo en motivos insistentes. Con desplazamientos en el escenario, se evidenció muy buen trabajo del dúo. En cuanto a formación de trío, vimos la obra de Hernán Ramírez Ávila, Trío con viola, op. 165 (2015), para clarinete, viola y piano. De carácter sombrío y espíritu dramático, presentó un buen desarrollo y adecuada combinación entre los instrumentos. De exigente ejecución, fue bien realizada por los intérpretes. 
Centrándonos en las obras solistas, hubo variada opción en esta versión. Interesante fue el trabajo de Sebastián Molina Villarroel, estudiante de composición que ya había sido seleccionado con una obra sinfónica en 2015. Su Fantasía para cello solo (2017) fue muy bien interpretada por Celso López, con pasajes aleatorios, sonidos percutidos y alturas indefinidas, organizada a base de notas tenidas tensionadas por ornamentos, que se tornan obsesivos en un movimiento perpetuo. Del cubano residente en Dinamarca, Louis Franz Aguirre se presentó Ibeyi-Añá (2006/2016), para flauta baja amplificada. De lenguaje contemporáneo, incluyó acciones de la voz, multifónicos, cuartos de tono y técnicas extendidas, intercalando respiraciones con líneas evocativas. De sonoridades sutiles en dinámicas suaves, generó amplitud sonora.

Para solista en multipercusión fue Resolana (2016), del venezolano Luis Ernesto Gómez. De tres secciones, la primera fue enérgica, la segunda lírica, tranquila y la tercera retomó el carácter rítmico. Resultó algo estática cada parte. Situación similar ocurrió con Túneles (2002) para saxofón tenor de Rodrigo Cádiz. Con multifónicos, slaps y otros recursos, combinó diversos materiales para construir una sumativa horizontal, de resultado algo estático en registros parejos. De Nicolás Kliwadenko Mouat escuchamos Didascalia (2013-2017), para flauta traversa, conformada por catorce momentos de notación aleatoria, donde el intérprete debió escoger el orden de ejecución. Construida por dos composiciones independientes realizadas por el intérprete de manera simultánea, se sustentó en el material sonoro de lenguaje contemporáneo presentado en partículas combinadas al interior de su forma fragmentaria.

Para guitarra presenciamos dos obras, una de ellas Quilla (2016), del mexicano Alan Sánchez. Basada en un motivo de 4 notas, apareció algo descontextualizada en el marco del festival por su lenguaje tonal y técnica guitarrística tradicional. Del chileno Felipe Pinto d'Aguiar Montt, Dicho Sea de Paso (2016), donde elementos de la guitarra flamenca son aislados y recontextualizados, utilizó scordatura y armónicos buscando un cierto minimalismo poético. Su escritura fragmentada a ratos hizo decaer el discurso por construir melodías de notas muy espaciadas, distantes.

Respecto de las obras con dispositivo electrónico, fueron solo dos las que utilizaron la tecnología en su elaboración sonora. Una fue Ludikalis 01 (2017), del académico DMUS Andrés Ferrari Gutiérrez. Presentado como un videojuego para clarinete, joystick, electrónica e imagen digital, resultado de sus investigaciones de posgrado, es descrito por su autor como una performance, lo que responde a dudas respecto del valor musical por sí solo. El total de la obra incorpora una puesta en escena donde se ponen "en juego", la "vida-opresor" con la "vida-músico", mediante sonidos electrónicos en tiempo real combinados con giros característicos de los videojuegos e interrupciones de parte del oponente, el clarinete.

Otra obra que incorporaba un dispositivo visual -microcámara y sistema de iluminación por leds para transmisión en tiempo real en una pantalla- no contó con todos los requerimientos técnicos. Así, Internas (2007) para flauta baja, del argentino Luciano Giambastiani, solo presentó la parte de iluminación. Con indicaciones gráficas, amplio uso de técnicas extendidas y secciones de improvisación, comenzó con iluminación leve para luego de una extensa sección oscurecerse por completo ante la mirada dubitativa del público.

Obra que requirió microfonía, condensadores y otros recursos para amplificación fue Estudio en azul (2004), para guitarra amplificada del ecuatoriano Juan Campoverde. De material mayoritariamente percutido por la mano izquierda con sonidos no convencionales de la mano derecha en volúmenes bajos, notación compleja y muy breve duración, generó expectativas por su preparación tecnológica que luego no se vieron satisfechas.

Finalmente y con efectivo uso de los dispositivos, del colombiano norteamericano César Potes presenciamos Surri Bachra Noscia: Homage (2016), para violín y electrónica en tiempo real. Su título, combinación entre Salvatore Sciarrino, J.S. Bach y un canto Baqara Sura, 
tributa al uso y tratamiento del violín por ambos compositores, junto con la riqueza tímbrica y microtonal de la recitación. En cinco escenas de escritura y técnicas contemporáneas, recurrió a diversas articulaciones y timbres. Con delicados pasajes de armónicos, suavemente dramática y la electrónica en octofonía, muy bien ejecutada por David Núñez Áñez.

Revisando las obras que incluyeron voz, bajó levemente el número con solamente tres obras, todas para pequeño ensamble. Una fue Nocturno (1968), por el Ensamble DMUS, para voz contralto, clarinete, guitarra, cello y piano, de Miguel Letelier, quien habitualmente aportó al festival con composiciones centradas en la voz. Obra extensa, de carácter dramático y lenguaje más experimental que sus últimas composiciones, en una conformación poco habitual que se desarrolló en fragmentos, generando un discurso pausado en torno a la voz utilizada sin texto explícito.

Para voces contratenor y barítono junto a cuarteto de cuerdas, fue Estupor (2017), del licenciado de esta casa de estudios, Víctor Gutiérrez Cuiza, quien dirigió el ensamble Ficción. Con técnicas extendidas, combinó bien armónicos y pizzicati de las cuerdas con efectos vocales que derivaron en momentos de intensidad expresiva, donde la parte vocal era la desintegración de la palabra estupor. Interesante, entre los estrenos a destacar, calurosamente recibido por el público. Finalmente, el Taller de Música Contemporánea estuvo con Silencio la tierra va a dar a luz un árbol (2016) de Marcos Stuardo Concha, con texto de Vicente Huidobro, para voz contralto, flauta, clarinete, violín, cello y piano. Construida como una extensa frase que recorre toda la pieza, en una ondulante idea sonora articulada en función del canto, logró un buen empaste de la voz y los instrumentos. De densidad expresiva, funcionó bien en este género.

Llegamos al día de cierre, siempre esperado por la participación de la Orquesta Sinfónica Nacional de Chile, en esta oportunidad bajo la batuta de Alejandra Urrutia, en jornada que comenzó con el Concierto Fúnebre (1939) del alemán Karl Amadeus Hartmann. Obra dramática y de profunda expresividad que refleja la dificultad del periodo histórico en que fue compuesta, fue liderada por Héctor Viveros como violín solista en una notable interpretación, apoyado con fervor por la OSNCH con una intensa ejecución.

En el concurso de obras sinfónicas, fue seleccionado el joven Ignacio Salvo Palominos, con Dies Irae (2017) para gran orquesta. Con un leimotiv que la organiza, la obra representó una suerte de dies irae posmoderno, en donde el compositor aprovechó, en su oportunidad juvenil, de presentar una obra audaz. Exigiendo fortissimos extremos apoyados por una numerosa fila de bronces, hubo momentos en que la orquesta evidenció poco entusiasmo por los niveles sonoros que debió alcanzar la ejecución. Si bien la obra apareció por momentos poco prolija y de estilo indefinido, demostró buen trabajo del prometedor autor. A no dudar, siempre será beneficioso para los jóvenes compositores el decidirse a tomar riesgos en el camino.

Clausurando esta versión y recordando a Miguel Letelier, la OSNCH presentó su Suite Scapin. De 1964, entendemos que fue seleccionada por ser un "clásico" de este compositor. Sin embargo, asimismo parece haber sido escogida para llegar sin riesgos a un público amplio. Consideramos que se pudo haber optado por un trabajo más reciente de Letelier mostrando una gama más extensa de su numeroso catálogo. Más aún, en la obra escogida, la OSCH apareció poco motivada y desganada durante la ejecución.

Si se trata de describir el carácter de esta versión, podríamos hablar de un festival tenaz, perseverante, básicamente por la diversidad de dificultades que debió sortear su realización. Para esto hay razones precisas. En primer lugar, la no obtención del financiamiento del CNCA, situación que generó dificultades de programación, cambios en la logística y, por cierto, el esfuerzo de intérpretes frente a condiciones económicas poco favorables. En segundo lugar, la coincidencia de fechas con un evento nacional de magnitud, como fue la visita del papa Francisco a Chile. Esto derivó en un enrarecido ambiente, los primeros días, 
debido a parte de la ciudad de Santiago conmocionada con actividades de la visita papal, llegando incluso a considerarse la suspensión de una jornada. Esto puede ser considerado un error de producción, por no haber previsto un cambio de fecha. Ahora bien, pese a los temores, el público acudió numeroso y entusiasta.

Otra situación inesperada se presentó el día jueves, por un corte masivo de luz en la capital que derivó en rostros tristes y abatidos entre los organizadores por tantos inconvenientes. Nuevamente y a pesar de la razonable preocupación, el concierto pudo ser realizado en buenas condiciones. Destacamos en ese sentido, como cada año, la impecable y destacable labor de todo el equipo de producción y escenario desplegado cada día.

Actividad enmarcada en esta edición fue el lanzamiento del libro Conversaciones con Fernando García de Eduardo Carrasco Pirard, que reúne entrevistas al compositor y fue producido en conjunto entre el DMUS y la SCD. Se llevó a cabo el lunes 15 en la Academia de Bellas Artes con la presencia del compositor y Premio Nacional de Artes Musicales, Fernando García Arancibia.

En cuanto a lo musical, en esta versión se dejó de abusar de la amplificación, volviendo al sonido natural, lo que se agradece. Por otro lado se plantean dudas dirigidas principalmente a la labor del Comité Seleccionador. Una de ellas, saber si se buscó que esta versión fuera de línea reciente, dejando prácticamente fuera obras referentes del repertorio contemporáneo. Y queda resonando una interrogante más importante. Esto es, la razón de la casi inexistente presencia de mujeres compositoras, con solamente una entre las treinta y siete obras presentadas. En ambos casos nos preguntamos si el problema está en la selección realizada o en que simplemente no postularon más obras que respondieran a estas inquietudes. En este sentido, sería necesario revisar las modalidades de convocatoria, postulación o programación.

Respecto de la ausencia de ensambles internacionales por falta de financiamiento, en oportunidades se le ha dado amplia tribuna a invitados que no parecen justificar cabalmente el pergamino que se supone conlleva ser extranjero invitado, ya sea por ejecución o propuesta de repertorio. Esta vez vimos cómo conjuntos nacionales estables realizaron presentaciones de mejor nivel que lo anteriormente señalado. Algo para tener en cuenta antes de continuar abusando de la etiqueta de invitado internacional. El énfasis que se le dio a lo local, obligado o no, permitió que más del setenta por ciento de la programación correspondiera a compositores chilenos, lo que no solo redunda en datos, sino en la agradable constancia de haber oído un aumento de obras nacionales interesantes. Como para volver a entusiasmarse del esfuerzo que significa la continuación de este festival. 